\title{
AVALIAÇÃO DO ATRIBUTO INTEGRALIDADE POR ENFERMEIROS DA ATENÇÃO PRIMÁRIA DO MUNICÍPIO DE CAMPINA GRANDE, PARAÍBA
}

\author{
ASSESSMENT OF THE ATTRIBUTE INTEGRALITY BY NURSES OF \\ PRIMARY CARE INTHE CITY OF CAMPINA GRANDE, PARAÍBA
}

\author{
Rafaela Simão de Abrantes ${ }^{1}$ \\ Aline de Paula Rêgo Graciano Luz ${ }^{2}$ \\ Alba Rossana Vieira Costa ${ }^{3}$ \\ Priscilla Kelly Policarpo Falcão ${ }^{4}$
}

\begin{abstract}
RESUMO: OBJETIVO: avaliar a presença e extensão da integralidade da atenção nos serviços de APS no município de Campina Grande, Paraíba, na perspectiva dos enfermeiros. Métodos: estudo transversal e descritivo, com 88 enfermeiros atuantes nas unidades básicas de saúde. A integralidade foi avaliada pelo instrumento de avaliação da atenção primária (Primary Care Assessment Tool - PCATool). A coleta de dados ocorreu entre os meses de julho e dezembro de 2019. RESULTADOS: O desempenho da integralidade foi satisfatório, com média de 6,9 para serviços disponíveis e 8,18 para serviços prestados. No entanto, a análise individual dos itens demonstrou insuficiência na realização de determinados procedimentos pelas equipes. CONCLUSÃO: A integralidade está presente nos serviços avaliados, mas os desafios encontradosprecisam ser considerados pelas equipes e gestores para o aperfeiçoamento dos serviços.
\end{abstract}

Palavras chave: Atenção Primária à Saúde. Avaliação de Serviços de Saúde. Integralidade em Saúde. Profissionais de Saúde.

ABSTRACT: OBJECTIVE: to evaluate the presence and extent of comprehensive care in primary care services in the city of Campina Grande, Paraíba, from the

\footnotetext{
Mestranda em Saúde Pública pela Universidade Estadual da Paraíba (UEPB). E-mail: rafaelaabrantes19@gmail.com.

2 Mestranda em Saúde Pública pela Universidade Estadual da Paraíba (UEPB). E-mail: aline_grac@yahoo.com.br.

${ }^{3}$ Enfermeira Residente em UTI - COREMU/IMIP. E-mail: vieira.albarossana@gmail.com.

${ }^{4}$ Mestranda em Saúde Pública pela Universidade Estadual da Paraíba (UEPB).

E-mail:
} pripolicarpo@gmail.com. 
perspective of nurses. METHODS: cross- sectional and descriptive study, with 88 nurses working in basic health units. Comprehensiveness was assessed using the Primary Care Assessment Tool (PCATool). Data collection took place between July and December 2019. RESULTS: Comprehensive performance was satisfactory, with an average of 6.9 for available services and 8.18 for services provided. However, the individual analysis of the items showed insufficiency in the performance of certain procedures by the teams. CONCLUSION: Comprehensiveness is present in the evaluated services, but the challenges encountered need to be considered by the teams and managers toimprove the services.

Keywords: Primary Health Care. Health Services Evaluation. Integrality in Health. HealthProfessionals. 


\section{INTRODUÇÃO}

A Atenção Primária à Saúde (APS) deve assumir a base do sistema de saúde, cuja posição central garante o papel de coordenadora dos serviços, estando integrada a estes, garantindo a resolução dos problemas de saúde mais frequentes na população (STARFIELD 2002). Esse conceito de APS é reconhecido pelo Ministério da Saúde, o qual indica a avaliação da qualidade dos seus serviços por instrumentos específicos, dentre eles o Primary Care Assessment Tool (PCATool) (BRASIL, 2010).

O PCATool, desenvolvido na Johns Hopkins University, avalia a qualidade dos serviços de APS por meio da verificação da existência de atributos essenciais e derivados (SHI et al, 2001). Esse instrumento apresenta-se em três versões destinadas ao adulto, aos cuidadores de crianças e aos profissionais de saúde, tendo sido validado para o Brasil e nomeado de Instrumento de Avaliação da Atenção Primária (PCATool-Brasil). (HARZHEIM, 2000; HARZHEIM, 2006; HAUSER et al., 2013).

Um dos atributos essenciais avaliado pelo por esse intrumento é a integralidade, analisada no presente estudo. O conceito de integralidade abriga muitos sentidos, constituindo um dos princípios do Sistema Único de Saúde (SUS). No PCATool, versão profissionais, esseatributo se subdivide em serviços prestados e serviços disponíveis, abordando um conjunto deações que o serviço deve oferecer a fim de garantir uma atenção integral, incluindo atividades de prevenção e promoção da saúde, bem como procedimentos específicos realizados pelos profissionais (BRASIL, 2010).

Alguns estudos nacionais que avaliaram os atributos da APS encontraram resultados variáveis. Kamper et al (2019) verificou, com 8.234 médicos cubanos do Programa Mais Médicos, um desempenho satisfatório da integralidade em todas as regiões do país, sugerindo que a formação desses profissionais pode ter contribuídos para os resultados encontrados. Já um estudo realizado em Fortaleza, 
com 451 profissionais da APS, encontrou baixo desempenho desse atributo (ROLIM et al., 2019).

Ademais, os estudos nacionais que utilizaram o PCATool em sua maioria foram voltados para os usuários (D’ÁVILA et al., 2017), enquanto aqueles direcionados aos profissionais de saúde são mais escassos, sobretudo na região nordeste. Destaca-se ainda a relevância desses estudos, dado o contexto de mudanças no financiamento da APS em 2019, sendo a avaliação um dos indicadores para o repasse de recursos para os serviços (BRASIL, 2019).

Considerando o exposto, o presente estudo teve como objetivo avaliar a presença e extensão da integralidade da atenção nos serviços de APS no município de Campina Grande, Paraíba, na perspectiva dos enfermeiros.

\section{MÉTODOS}

Trata-se de um estudo descritivo, com abordagem quantitativa e delineamento transversal. A população do estudo foi composta pelos enfermeiros da APS, cujo total era de 117 profissionais, de acordo com dados atualizados do DATASUS (BRASIL, 2019). Desse total, 94 foram considerados elegíveis de acordo com os critérios estabelecidos. A amostra finalfoi composta por 88 enfermeiros que atuavam em 81 Unidades Básicas de Saúde da Família da zona urbana e rural de Campina Grande-PB. Foram incluídos os trabalhadores que atuavam hápelo menos seis meses nos serviços e com carga horária mínima de 20 horas semanais. Excluíram-se aqueles em período de licença ou férias na época em que foram realizadas as coletas.

Para a coleta dos dados foi utilizado um protocolo dividido em: 1- questões sociodemográficas, de formação, qualificação profissional e contexto de trabalho; 2avaliação da presença e extensão da integralidade da atenção pelo PCATool. Esse atributo é estruturado da seguinte forma:

- $\quad$ Integralidade - Serviços Disponíveis. Formado por 22 itens (E1 a E22): inclui questõessobre o escopo de procedimentos oferecidos pelo serviço. 
- Integralidade - Serviços Prestados. Formado por 15 itens (F1 a F15): abrange questõessobre uma gama de temas abordados pelos profissionais junto aos pacientes.

Cada questão apresenta respostas cujos valores variam em escala tipo Likert, sendo: $4=$ com certeza $\operatorname{sim}, 3=$ provavelmente $\operatorname{sim}, 2=$ provavelmente não, $1=\mathrm{com}$ certeza não e $9=$ não sei/nãolembro. Esses valores foram transformados em escala contínua de 0 a 10 por meio da fórmula: Escore de 0 a 10 da integralidade

$$
=\frac{(\text { escore de } 1 \mathrm{a} 4-1) \times 10}{3}
$$

Foram considerados satisfatórios os escores $\geq 6,6$ (Escore Alto), correspondendo às responstas maiores ou igual a 3 (provavelmente sim) na escala Likert. Dessa forma, os escoresforam dicotomizados em Escore Alto $(\geq 6,6)$ e Escore Baixo $(\leq 6,6)$.

A coleta ocorreu entre julho e dezembro de 2019, nas dependências físicas da UBS, por três pesquisadoras capacitadas, do Mestrado em Saúde Pública da Universidade Estadual da Paraíba. Para tal, foi utilizado o Manual do Instrumento de Avaliação da Atenção Primária, do MS. Os dados foram digitados em planilhas do Excel. Foi realizado o teste de Kolmogorov- Smirnov para verificação da distribuição das variáveis. $\mathrm{Na}$ análise descritiva, estas apresentaram-se na forma de média, conforme sua distribuição, além de frequências absolutas para cada item do PCATool.

Essa pesquisa seguiu as orientações da Resolução 466/2012 do Conselho Nacional de Saúde, tendo sido aprovada pelo Comitê de Ética em Pesquisa da Universidade Estadual da Paraíba por meio do parecer $n^{\circ} 3.251 .662$.

\section{RESULTADOS}

Participaram do estudo 88 enfermeiros atuantes nas Unidades Básicas de Saúde de Campina Grande, de um total de 117 profissionais. Destes, 94 foram elegíveis segundo os critérios de inclusão e exclusão. A taxa de perda foi de $6,38 \%$ 
$(n=6)$, incluindo recusas (3) e insucesso na aplicação do questionário após quatro tentativas (3).

A idade média foi de 42,26, com mediana de 40 anos. A amostra foi predominantementedo sexo feminino $(n=85,9,6 \%)$.

Quanto à formação e qualificação profissional, a maioria conclui a graduação em instituição pública $(69,3 \%)$, possuía pós graduação na área da APS $(87,5 \%)$, cuja especialização foi o tipo mais frequente $(71,6 \%)$ e já participaram de atividade de educação permanente. A média de tempo de formado foi de 17,41 anos.

No que se refere ao contexto de trabalho, $68,2 \%$ possuíam vínculo estatutário e $55,7 \%$ afirmaram não possuir ou desconhecer o incentivo municipal para realização de educação permanente. A mediana de tempo de atuação nos serviços de APS e de atuação na equipe foi de 15 e 6 anos, respectivamente.

A média dos escores da integralidade - serviços disponíveis e serviços prestados foi de 6,9 e 8,18 e a prevalência do escore geral alto foi de $53(60,2 \%)$ e $80(90,9)$, respectivamente. 
Tabela 1 - Distribuição das variáveis sociodemográficas, de contexto profissional e de trabalho dos enfermeiros das Unidades Básicas de Saúde no município de Campina Grande, Paraíba.

\begin{tabular}{|c|c|c|c|}
\hline Variável & n (\%) & Variável & n (\%) \\
\hline Sexo & & Vínculo empregatício & \\
\hline Feminino & $85(96,6)$ & Estatutário & $60(68,2)$ \\
\hline Masculino & $3(3,4)$ & Contrato & $28(31,8)$ \\
\hline Instituição & & Incentivo municipal & \\
\hline Pública & $61(69,3)$ & Sim & $39(44,3)$ \\
\hline Privada & $27(30,7)$ & Não/Não sei & $49(55,7)$ \\
\hline Pós graduação na APS & & Variável & Média*/Mediana† \\
\hline Sim & $77(87,5)$ & Idade & $40 \dagger$ \\
\hline Não & $11(12,5)$ & Tempo de formado & $17,41^{*}$ \\
\hline Tipo de pós graduação & & $\begin{array}{c}\text { Tempo de atuação na } \\
\text { APS }\end{array}$ & $15 t$ \\
\hline Nenhuma & $11(12,5)$ & $\begin{array}{c}\text { Tempo de atuação na } \\
\text { equipe }\end{array}$ & $6 \dagger$ \\
\hline Especialização & $63(71,6)$ & & \\
\hline $\begin{array}{c}\text { Mestrado/doutorado } \\
\text { Educacão Permanente }\end{array}$ & $14(15,9)$ & & \\
\hline Sim & $73(83)$ & & \\
\hline Não & $15(17)$ & & \\
\hline
\end{tabular}

Tabela 2 - Distribuição dos escores alto e baixo segundo os enfermeiros das Unidades Básicas de Saúde do município de Campina Grande, Paraíba.

\begin{tabular}{lcccc}
\hline Atributo & $\begin{array}{c}\text { EscoreAlto } \\
\mathbf{n}(\%)\end{array}$ & $\begin{array}{c}\text { EscoreBaixo } \\
\mathbf{n}(\%)\end{array}$ & Média & DP \\
\hline Integralidade - Serviços Disponíveis & $53(60,2)$ & $35(39,8)$ & 6,9 & 1,32 \\
Integralidade - Serviços Prestados & $80(90,9)$ & $8(9,1)$ & 8,18 & 1,18 \\
\hline
\end{tabular}


Tabela 3 - Distribuição da frequência das respostas de cada item da integralidade segundo os enfermeiros das Unidades Básicas de Saúde do município de Campina Grande, Paraíba.

\begin{tabular}{|c|c|c|c|c|}
\hline \multirow[t]{2}{*}{$\begin{array}{l}\text { Integralidade - Serviços } \\
\text { Disponíveis }\end{array}$} & $\begin{array}{l}\text { Com certeza } \\
\text { sim }\end{array}$ & $\begin{array}{l}\text { Provavelmente } \\
\text { sim }\end{array}$ & $\begin{array}{l}\text { Provavelmente } \\
\text { não }\end{array}$ & $\begin{array}{l}\text { Com } \\
\text { certeza } \\
\text { não }\end{array}$ \\
\hline & $n(\%)$ & $\mathrm{n}(\%)$ & n (\%) & $\mathrm{n}(\%)$ \\
\hline Aconselhamento nutricional & $54(61,4)$ & $22(25)$ & $4(4,5)$ & $8(9,1)$ \\
\hline Imunizações & $77(87,5)$ & $5(5,7)$ & $3(3,4)$ & $3(3,4)$ \\
\hline $\begin{array}{l}\text { Verificação se as famílias } \\
\text { podem participar de algum } \\
\text { benefício de assistência social }\end{array}$ & $61(69,3)$ & $15(17)$ & $11(12,5)$ & $1(1,1)$ \\
\hline Avaliação da saúde bucal & $54(61,4)$ & $6(6,8)$ & $6(6,8)$ & $22(25)$ \\
\hline Tratamento dentário & $24(27,3)$ & $9(10,2)$ & $8(9,1)$ & $24(27,3)$ \\
\hline $\begin{array}{l}\text { Planejamento familiar ou } \\
\text { métodos anticoncepcionais }\end{array}$ & $78(88,6)$ & $9(10,2)$ & $1(1,1)$ & - \\
\hline $\begin{array}{l}\text { Aconselhamento } \\
\text { tratamento para o ou } \\
\text { prejudicial de drogas (lícitas ou } \\
\text { ilícitas). }\end{array}$ & $40(45,5)$ & $33(37,5)$ & $9(10,2)$ & $6(6,8)$ \\
\hline $\begin{array}{l}\text { Aconselhamento para } \\
\text { problemas de saúde mental }\end{array}$ & $55(62,5)$ & $22(25)$ & $9(10,2)$ & $2(2,3)$ \\
\hline $\begin{array}{l}\text { Sutura de um corte que } \\
\text { necessite de pontos }\end{array}$ & $13(14,8)$ & $1(1,1)$ & $12(13,6)$ & $62(70,5)$ \\
\hline $\begin{array}{l}\text { Aconselhamento e solicitação } \\
\text { de teste anti-HIV }\end{array}$ & $78(88,6)$ & $6(6,8)$ & $1(1,1)$ & $3(3,4)$ \\
\hline $\begin{array}{l}\text { Identificação (Algum tipo de } \\
\text { avaliação) de problemas } \\
\text { auditivos (para escutar). }\end{array}$ & $34(38,6)$ & $26(29,5)$ & $11(12,5)$ & $17(19,3)$ \\
\hline $\begin{array}{l}\text { Identificação (Algum tipo de } \\
\text { avaliação) de problemas } \\
\text { visuais (para enxergar) }\end{array}$ & $30(34,1)$ & $26(29,5)$ & $17(19,3)$ & $15(17)$ \\
\hline $\begin{array}{l}\text { Colocação de tala (ex: para } \\
\text { tornozelo torcido) }\end{array}$ & $7(8)$ & $4(4,5)$ & $14(15,9)$ & $63(71,6)$ \\
\hline Remoção de verrugas & $6(6,8)$ & $1(1,1)$ & $11(12,5)$ & $70(79,5)$ \\
\hline $\begin{array}{l}\text { Exame preventivo para câncer } \\
\text { de colo de útero (Teste } \\
\text { Papanicolau) }\end{array}$ & $83(94,3)$ & $5(5,7)$ & - & - \\
\hline $\begin{array}{l}\text { Aconselhamento sobre como } \\
\text { parar de fumar }\end{array}$ & $59(67)$ & $21(23,9)$ & $4(4,5)$ & $4(4,5)$ \\
\hline Cuidados pré-natais & $86(97,7)$ & $2(2,3)$ & - & - \\
\hline Remoção de unha encravada & $7(8)$ & $5(5,7)$ & $15(17)$ & $61(69,3)$ \\
\hline $\begin{array}{l}\text { Orientações sobre cuidados } \\
\text { em saúde caso o paciente } \\
\text { fique incapacitado }\end{array}$ & $40(45,5)$ & $30(34,1)$ & $7(8)$ & $11(12,5)$ \\
\hline $\begin{array}{l}\text { Aconselhamento sobre as } \\
\text { mudanças que acontecem } \\
\text { com o envelhecimento }\end{array}$ & $58(65,9)$ & $28(31,8)$ & - & $2(2,3)$ \\
\hline $\begin{array}{l}\text { Orientações sobre cuidados no } \\
\text { domicílio para alguém da } \\
\text { família do paciente }\end{array}$ & $75(85,2)$ & $11(12,5)$ & - & $2(2,3)$ \\
\hline Inclusão em programa de & $28(43,2)$ & $16(18,2)$ & $15(17)$ & $19(21,6)$ \\
\hline
\end{tabular}




\begin{tabular}{|c|c|c|c|c|}
\hline \multicolumn{5}{|l|}{$\begin{array}{l}\text { suplementação alimentar (ex: } \\
\text { leite) }\end{array}$} \\
\hline \multirow[t]{2}{*}{$\begin{array}{l}\text { Integralidade - Serviços } \\
\text { Prestados }\end{array}$} & $\begin{array}{l}\text { Com certeza } \\
\text { sim }\end{array}$ & $\begin{array}{l}\text { Provavelmente } \\
\text { sim }\end{array}$ & $\begin{array}{l}\text { Provavelmente } \\
\text { não }\end{array}$ & $\begin{array}{l}\text { Com } \\
\text { certeza } \\
\text { não }\end{array}$ \\
\hline & n (\%) & n (\%) & n (\%) & n (\%) \\
\hline $\begin{array}{l}\text { Conselhos sobre alimentação } \\
\text { saudável ou sobre dormir } \\
\text { suficientemente }\end{array}$ & $78(88,6)$ & $10(11,4)$ & - & (1) \\
\hline $\begin{array}{l}\text { Segurança no lar, ex: como } \\
\text { guardar medicamentos em } \\
\text { segurança }\end{array}$ & $62(70,5)$ & $25(28,4)$ & - & $1(1,1)$ \\
\hline $\begin{array}{l}\text { Aconselhamento sobre o uso } \\
\text { de cinto de segurança e outros } \\
\text { relacionados }\end{array}$ & $30(34,1)$ & $31(35,2)$ & $23(26,1)$ & $4(4,5)$ \\
\hline $\begin{array}{l}\text { Maneiras de lidar com conflitos } \\
\text { de família }\end{array}$ & $40(45,5)$ & $40(45,5)$ & $5(5,7)$ & $3(3,4)$ \\
\hline $\begin{array}{l}\text { Conselhos a respeito de } \\
\text { exercícios físicos apropriados }\end{array}$ & $66(75)$ & $19(21,6)$ & $1(1,1)$ & $2(2,3)$ \\
\hline $\begin{array}{l}\text { Testes de sangue para } \\
\text { verificar os níveis de colesterol }\end{array}$ & $68(77,3)$ & $11(12,5)$ & $3(3,4)$ & $6(6,8)$ \\
\hline $\begin{array}{l}\text { Verificar e discutir } \text { os } \\
\text { medicamentos que o paciente } \\
\text { está usando }\end{array}$ & $72(81,8)$ & $16(18,2)$ & - & - \\
\hline $\begin{array}{l}\text { Possíveis exposições a } \\
\text { substâncias perigosas no lar, } \\
\text { no trabalho etc }\end{array}$ & $41(46,6)$ & $34(38,6)$ & $11(12,5)$ & $2(2,3)$ \\
\hline $\begin{array}{l}\text { Pergunta se o paciente tem } \\
\text { uma arma de fogo e orienta } \\
\text { como guardá-la }\end{array}$ & $8(9,1)$ & $8(9,1)$ & $31(35,2)$ & $41(46,6)$ \\
\hline $\begin{array}{l}\text { Como prevenir queimaduras } \\
\text { causadas por água quente, } \\
\text { óleo quente }\end{array}$ & $44(50)$ & $32(36,4)$ & $9(10,2)$ & $3(3,4)$ \\
\hline Como prevenir quedas & $56(63,6)$ & $30(34,1)$ & $2(2,3)$ & - \\
\hline $\begin{array}{l}\text { Prevenção de osteoporose em } \\
\text { mulheres }\end{array}$ & $49(55,7)$ & $30(34,1)$ & $9(10,2)$ & - \\
\hline $\begin{array}{lcc}\text { Cuidado de problemas } \\
\text { comuns relativos à } \\
\text { menstruação ou a menopausa }\end{array}$ & $75(85,2)$ & $12(13,6)$ & $1(1,1)$ & - \\
\hline $\begin{array}{l}\text { Maneiras de lidar com os } \\
\text { problemas de comportamento } \\
\text { das crianças }\end{array}$ & $61(69,3)$ & $24(27,3)$ & $2(2,3)$ & $1(1,1)$ \\
\hline $\begin{array}{l}\text { Mudanças do crescimento e } \\
\text { desenvolvimento da criança } \\
\text { por cada faixa }\end{array}$ & $77(87,5)$ & $10(11,4)$ & $1(1,1)$ & - \\
\hline
\end{tabular}

\section{DISCUSSÃO}

Para que a integralidade seja alcançada, a atenção primária deve ser capaz de reconheceras necessidades mais frequentes relacionadas à saúde da população e 
disponibilizar recursos para atendê-las. Tais necessidades podem ser sintomas, desconfortos, enfermidades ou intervenções de prevenção e promoção de saúde. Serviços limitados em extensão e profundidade implica aumento de morbidades e agravos evitáveis (STARFIELD, 2002).

De modo geral, o presente estudo verificou um desempenho satisfatório do atributo integralidade, embora com variações nos escores dos componentes serviços prestados $(8,18)$ e serviços disponíveis $(6,9)$, este último próximo ao ponto de corte de 6,6.

Desempenhos satisfatórios também foram encontrados em estudos realizados em São Paulo (GOMES et al., 2018; MARTINS et al., 2016), Rio Grande do Sul (PENSO et al., 2017), e Goiás (OLIVEIRA et al., 2016). Já um estudo realizado em Fortaleza, encontrou escores insatisfatórios tanto para o componente serviços prestados quanto para serviços disponíveis na avaliação por enfermeiros (ROLIM et al., 2019).

Quanto ao perfil dos profissionais, foram encontrados estudos com resultados semelhantes, com amostra predominantemente do sexo feminino e com formação em instituição pública (FERREIRA et al., 2016; OLIVEIRA et al., 2016; ROLIM et al., 2019). Além disso, a média da idade (HAUSER et al., 2013; OLIVEIRA et al., 2016; ROLIM et al., 2019) e tempo de formado (FERREIRA et al., 2016; ROLIM et al., 2019) desses estudos também foram próximos ao verificados nesta pesquisa.

No que se refere à qualificação profissional, os resultados convergem com o verificados em outros estudos, cuja maioria dos profissionais possuíam pós graduação na área da APS, sendo a especialização a mais frequente (CHOMATAS et al., 2013; ROLIM et al., 2019; MAIAet al., 2020). Estudo realizado no Distrito Federal encontrou que essas especializações impactavam na melhoria da competência profissional, resultando em novos modos de agir entre os profissionais da APS (SCHERER et al., 2016). Não foi possível a comparação quanto à educação permanente, visto que não foram encontrados estudos avaliativos com o PCATool que verificaram essa variável.

Em relação ao contexto de trabalho, também foram verificados resultados similares quanto ao vínculo estatutário (FERREIRA et al., 2016; NASCIMENTO et al., 2016; OLIVEIRA et al., 2016). Diferentemente, o incentivo municipal à educação 
permanente ou continuada apresentou prevalências positivas em outros estudos (CHOMATAS et al., 2013; OLIVEIRA et al., 2016). O resultado do presente estudo indica um baixo grau de envolvimento da gestão com essas ações ou estas não têm sido suficientes para serem percebidas pelos profissionais, reforçando a necessidade de sua implantação. Já o tempo de atuação na APS e na equipe foi inferior ao verificado em outros estudos (OLIVEIRA et al., 2016; FERREIRA et al., 2016; NASCIMENTO et al., 2016).

A verificação das questões individuais da avaliação do atributo integralidade revelou que, apesar da maioria ter apresentado elevadas prevalências positivas, alguns itens relevantes mostraram-se negativamente avaliados. Nos serviços disponíveis, isso foi verificado nos itens que tratam sobre a avaliação de saúde bucal e a realização de tratamento dentário, além de procedimentos como realização de sutura, colocação de tala e remoção de verrugas.

De fato, o município de Campina Grande possui uma cobertura de equipes de saúde bucal vinculadas às equipes de saúde da família de apenas 43,18\% (BRASIL, 2020), o que explicaria a realização insuficiente das ações e procedimentos nesse âmbito. Além disso, a própria situação organizacional do município, caracterizada pelo deslocamento de serviços odontológicos das unidades básicas para os centros de saúde, dificulta o alcance da integralidade, visto que cria barreiras para a continuidade do cuidado ao paciente pela mesma equipe.

Já a realização de suturas, colocação de tala e remoção de verrugas são procedimentos que exigem, além de habilidades dos profissionais, condições mínimas tanto no fornecimento de materiais e insumos quanto em questões estruturais. Um estudo realizado no mesmo município em 2018, verificou que tais condições foram consideradas inadequadas pelos profissionais das unidades básicas de saúde (PEDRAZA et al., 2018). Tal situação reflete uma realidade nacional e afeta diretamente o alcance de uma atenção integral (BOUSQUAT et al., 2017).

Na avaliação de serviços prestados, a prevalência negativa esteve presente no item quetrata do conhecimento sobre a posse de arma de fogo pelo paciente e se é feita orientação sobre como guardá-la. Isso pode ser explicado pela timidez ou receio do profissional para abordar essa questão, sendo necessárias estratégias de 
comunicação menos invasivas para divulgação dessas informações, considerando a relevância da temática.

Apesar das respostas negativas descritas acima, houve o predomínio de avaliações positivas, demonstrando um potencial das equipes para o alcance da integralidade. Isso pode ser observado em serviços como cuidados pré-natais, exame preventivo para câncer de colo de útero, planejamento familiar e métodos anticoncepcionais, conselhos sobre alimentação saudável e conhecimento acerca dos medicamentos usados pelos pacientes, os quais não apresentaram nenhuma resposta negativa.

\section{CONCLUSÃO}

O atributo integralidade apresentou avaliação satisfatória pelos enfermeiros de CampinaGrande com predominância de avaliações positivas indicando um potencial das equipes nesses serviços. Apesar disso, a verificação individual dos itens demonstrou insuficiência na realizaçãode determinados procedimentos clínicos pelas equipes, incluindo a atenção odontológica. Tais achados implicam desafios a serem enfrentados para o alcance de uma atenção integral.

As deficiências detectadas são passíveis de mudanças através de iniciativas internas aoprocesso de trabalho das equipes e por parte dos gestores, com garantia de estrutura física mínima, apoio às equipes na organização dos serviços e capacitação orientada pelas dificuldades enfrentadas pelos enfermeiros.

Apesar de tratar-se de um estudo local, os resultados encontrados podem ser utilizados pelos profissionais e gestores do município, orientando iniciativas futuras que busquem o aperfeiçoamento dos serviços. 


\section{REFERÊNCIAS BIBLIOGRÁFICAS}

BOUSQUAT A. et al. Tipologia da estrutura das unidades básicas de saúde brasileiras: os $5 \mathrm{R}$. Cad. Saúde Pública, Rio de Janeiro, v.33, n.8, e00037316, 2017. Disponível em: https://doi.org/10.1590/0102-311x00037316. Acesso em: 15 jan. de 2020.

Brasil. Ministério da Saúde. Manual do instrumento de avaliação da atenção primária à saúde: Primary Care Assessment Tool PCAToolBrasil - Brasil. Secretaria de Atenção à Saúde, Departamento de Atenção Básica. 1 ed. Brasília: MS, 2010. ISBN 978-85-334-1696-3. Disponível em: http://bvsms.saude.gov.br/bvs/publicacoes/manual_avaliacao_pcatool_brasil.pdf. Acesso em: 10 jan. 2020.

$\begin{array}{llll}\text { Brasilia } & \text { Ministério da Saúde, Datasus. Cadastro Nacional de Estabelecimentos de Saúde. } \\ \text { (DF); } & 2019 . & \text { Disponível } & \text { em: }\end{array}$ http://tabnet.datasus.gov.br/cgi/tabcgi.exe?cnes/cnv/equipepb.def. Acessoem: 10 jul 2019.

. Ministério da Saúde, e-Gestor Atenção Básica, Informação e Gestão da Atenção Básica. Brasília (DF);2020. Disponível em: https://egestorab.saude.gov.br/paginas/acessoPublico/relatorios/relHistoricoCoberturaSB.xhtml;js essio nid=QYY4zKOg6dXrbEou+OnOFOxq. Acesso em: 19 nov. 2019.

Ministério da Saúde. Portaria $n^{\circ} 3.222$ de 19 de dezembro de 2019. Dispõe sobre os indicadores do pagamento por desempenho, no âmbito do Programa Previne Brasil. Diário Oficial daUnião. Brasília, p. 172, 11 Dez 2019. Disponível em: http://www.in.gov.br/en/web/dou//portaria-n- 3.222-de-10-de-dezembro-de-2019-232670481. Acesso em: 15 jan. 2020.

CHOMATAS, E. et al. Avaliação da presença e extensão dos atributos da atenção primária em Curitiba. Rev. Bras. Med. Fam. e Comunidade, Rio de Janeiro, v. 8, n. 29, p. 294-303, 2013. Disponível em: https://doi.org/10.5712/rbmfc8(29)828. Acesso em: 20 jan. 2020.

D'AVILA, O.P. et al. O uso do Primary Care Assessment Tool (PCAT): uma revisão integrativa e proposta de atualização. Ciênc. Saúde Coletiva, Rio de Janeiro, v. 22, n. 3, p. 855-865, 2017. Disponivel em: http://www.scielo.br/scielo.php?script=sci_arttext\&pid=S0102311X2006000800013.Acesso em: 11 jan. 2020.

FERREIRA, V. D. et al. Avaliação dos atributos da Atenção Primária à Saúde em um Município Mineiro. Esc Anna Nery, Rio De Janeiro, v. 20, n. 4: e20160104, 2016a. Disponível em: http://www.scielo.br/pdf/ean/v20n4/1414-8145-ean-20-04-20160104.pdf. Acesso em: 15 jan. 2020.

GOMES, M. F. P; FRACOLLI, L. A. Avaliação da estratégia saúde da família sob a ótica dos profissionais. Rev Bras Promoç Saúde, Fortaleza, v. 31, n. 3, p. 1-13, 2018. Disponível em: DOI:10.5020/18061230.2018.7108. Acesso em: 16 jan. 2019.

HARZHEIM, E. et al. Consistência interna e confiabilidade da versão em português do Instrumento de Avaliação da Atenção Primária (PCATool-Brasil) para serviços de saúde infantil. Cad Saúde Pública, Rio de Janeiro, v. 22, n. 8, p. 1649-1659, 2006. Disponível em: http://www.scielo.br/scielo.php?script=sci_arttext\&pid=S0102-311X2006000800013. Acesso em: 06jan. 2020.

HARZHEIM, E. et al. Quality and effectiveness of different approaches to primary care delivery in Brazil. BMC Health Services Research, London, v. 6, n. 156, p. 1-13, 2000. Disponível em: DOI 10.1186/1472-6963-6-156. Acesso em: 11 jan. 2020. 
HAUSER, L. et al. Tradução, adaptação, validade e medidas de fidedignidade do Instrumento de Avaliação da Atenção Primária à Saúde (PCATool-Brasil) no Brasil: versão profissionais de saúde. Rev. Bras. Med. Fam. Comunidade, Rio de Janeiro, v. 8 n. 29 p. 244-55, 2013. Disponível em: http://dx.doi.org/10.5712/rbmfc8(29)821. Acesso em: 11 jan. 2019.

KEMPER, E. Avaliação da qualidade da Atenção Primária no Programa Mais Médicos. APS EM REVISTA, Minas Gerais, v. 1, n. 1, p. 75-87, 2019. Disponível em: https://doi.org/10.14295/aps.v1i1.17. Acesso em: 20 jan 2020.

MAIA, L. G. et al. A qualidade de serviços de atenção primária, a formação profissional e o Programa Mais Médicos em uma região de saúde do sudoeste goiano. Rev. bras. epidemiol., Rio de Janeiro, v. 23, e200014, 2020. Disponível em: http://www.scielo.br/scielo.php?script=sci_arttext\&pid=S1415-

790X2020000100413\&lng=en\&nrm=iso. Acesso em: 20 jan. 2020.

MARTINS, J. S. et al. Comparative Study between Health Care Units with and without Family Health Strategy using PCATool. Rev. Bras. Med. Fam. Comunidade, Rio de Janeiro, v. 11, n. 38, p. 1-13, 2016. Disponível em: https://doi.org/10.5712/rbmfc11(38)1252. Acesso em: 20 jan. 2020.

NASCIMENTO, L. do et al. Qualidade da Atenção Primária à Saúde de crianças e adolescentes vivendo com HIV. Rev Latino-Am Enfermagem, Ribeirão Preto, v. 24, n. e2720, p. 1-10, 2016. Disponível em: http://www.scielo.br/pdf/rlae/v24/pt_0104-1169-rlae-24-02720.pdf. Acesso em: 16 jan. 2020.

OLIVEIRA, M.P. R.; MENEZES, I. H. C. F.; SOUSA, L. M.; PEIXOTO, M. R. G. Formação e qualificação de profissionais de saúde: fatores associados à qualidade da atenção primária. Rev Bras Educ Med, Rio de Janeiro, v. 40, n. 4, p. 547-59, 2016. Disponível em: http://www.scielo.br/pdf/rbem/v40n4/1981-5271-rbem-40-4-0547.pdf. Acesso em: 15 jan. 2020.

PEDRAZA P. F. et al. Characterization of work of nurses and health professionals in Support Centerfor Family Health in Primary Health Care. ABCS Health Sciences, São Paulo, v. 43, n. 3, p.77-83, 2018. Disponível em: http://dx.doi.org/10.7322/abcshs.v43i2.993. Acesso em: 16 jan. 2020.

PENSO, J. M. et al. Avaliação da Atenção Primária à Saúde utilizando o Instrumento PCAToolBrasil. Rev. Bras. Med. Fam. Comunidade, v. 12, n. 39, p. 1-9, 22 jun. 2017. Disponível em: https://doi.org/10.5712/rbmfc12(39)1212. Acesso em: 15 jan. 2020.

ROLIM, L.B. et al. Evaluation of Primary Health Care attributes of Fortaleza city, Ceará State, Brazil. Rev Bras Enferm. v. 72, n. 1, p. 19-26, 2019. Disponível em: https://doi.org/10.1590/0034 -7167-2018. Acesso em: 15 nov. 2019.

SCHERER, M. D. A. et al. Cursos de especialização em Saúde da Família: o que muda no trabalho com a formação? Interface (Botucatu), Botucatu, v. 20, n. 58, p. 691-702, 2016. Disponível em: http://www.scielo.br/scielo.php?script=sci_arttext\&pid=S1414$32832016000300691 \&$ Ing=en\&nrm=iso. Acesso em: 30 jan 2020. SHI, L; STARFIELD, B.; XU, J. Validating the Adult Primary Care Assessment Tool. Fam. Pract., Reino Unido, v. 50, n. 2, p. 161-175, 2001. Disponível em: https://www.jhsph.edu/research/centers- and-institutes/johnshopkins-primary-care-policy-center/PCAT\%20pubs/Shi\%202001.pdf. Acesso em: 11 jan. 2020.

STARFIELD, B. Atenção primária: equilíbrio entre necessidades de saúde, serviços e tecnologia.Brasília: UNESCO, 2002. ISBN: 85-87853-72-4. 